\title{
A Deep Multiwavelength View of Binaries in $\omega$ Centauri
}

\author{
Daryl Haggard ${ }^{*}$, Adrienne M. Cool ${ }^{\dagger}$, Tersi Arias ${ }^{\dagger}$, Michelle B. Brochmann ${ }^{\dagger}$, Jay \\ Anderson** and Melvyn B. Davies \\ ${ }^{*}$ Center for Interdisciplinary Exploration and Research in Astrophysics, Northwestern University, 2145 \\ Sheridan Road, Evanston, IL 60208, USA; dhaggard@northwestern.edu \\ ${ }^{\dagger}$ Department of Physics and Astronomy, San Francisco State University, 1600 Holloway Avenue, San Francisco, \\ CA 94132, USA \\ ${ }^{* *}$ Space Telescope Science Institute, Baltimore, MD 21218, USA \\ ${ }^{\ddagger}$ Lund Observatory, Box 43, SE-221 00 Lund, Sweden
}

\begin{abstract}
.
We summarize results of a search for X-ray-emitting binary stars in the massive globular cluster $\omega$ Centauri (NGC 5139) using Chandra and HST. ACIS-I imaging reveals 180 X-ray sources, of which we estimate that $45-70$ are associated with the cluster. We present 40 identifications, most of which we have obtained using ACS/WFC imaging with HST that covers the central $10^{\prime} \times 10^{\prime}$ of the cluster. Roughly half of the optical IDs are accreting binary stars, including 9 very faint blue stars that we suggest are cataclysmic variables near the period limit. Another quarter comprise a variety of different systems all likely to contain coronally active stars. The remaining 9 X-ray-bright stars are an intriguing group that appears redward of the red giant branch, with several lying along the anomalous RGB. Future spectroscopic observations should reveal whether these stars are in fact related to the anomalous RGB, or whether they instead represent a large group of "sub-subgiants" such as have been seen in smaller numbers in other globular and open clusters.
\end{abstract}

Keywords: binaries: close - color-magnitude diagrams (HR diagram) - globular clusters: individual ( $\omega$ Centauri) — novae, cataclysmic variables — stars: neutron, white dwarfs - X-rays: binaries

PACS: $95,97,98$

\section{INTRODUCTION}

Globular clusters (GCs) host a variety of binary star systems, formed both primordially and dynamically via stellar encounters. These binaries play a crucial role in the dynamical evolution of GCs, providing an energy reservoir that can delay core collapse for many times the half-mass relaxation time [e.g., 1]. The dense cluster environment also dramatically alters the evolution of GC binaries [e.g., 2, 3, 4, 5]. X-ray-emitting systems have, in particular, emerged as a promising source of information about the history of binary formation and destruction in galactic GCs.

The Chandra X-ray Observatory's high spatial resolution and resulting sensitivity to point sources makes it possible to obtain nearly complete samples of compact accreting binaries in nearby globular clusters. The ability to pinpoint sources to $<1^{\prime \prime}$ also means that the stars responsible for the X-ray emission may be recovered at other wavelengths even in the crowded fields of GCs. While the high luminosity X-ray binaries $\left(L_{x}=10^{36-38} \mathrm{erg} \mathrm{s}^{-1}\right)$ are understood to be accreting neutron stars $[6,7,8]$, the low X-ray-luminosity sources are now known to comprise several distinct populations: cataclysmic variables (CVs), quiescent neutron stars (qNS, or qLMXB), millisecond pulsars (MSPs), and binaries with chromospherically active stars, i.e. active binaries [ABs; 9, 10, 11, 12]. Of these, only the quiescent NSs, with their distinctive soft X-ray spectra, can be identified uniquely on the basis of X-ray observations alone [13, 14]. For others, optical (or radio, in the case of MSPs) follow-up is essential.

$\omega$ Cen is the most massive GC in the Milky Way $\left[4 \times 10^{6} \mathscr{M}_{\odot} ; 15\right]$. At $4.9 \mathrm{kpc}$, it is relatively nearby, making it possible to detect low-luminosity X-ray sources in modest exposure times with Chandra. Its unusually complex stellar populations have prompted debate as to whether $\omega$ Cen is a GC at all - it is instead likely to be the remnant of a dwarf galaxy accreted by the Milky Way [16, 17, 18, 19] — and controversy continues over the existence of an intermediate black hole in its core, e.g. see Noyola et al. [20] vs. Anderson and van der Marel [21]. Regardless of $\omega$ Cen's origins, the binary stars that it contains play a crucial role in its dynamical evolution and can in turn shed light on the impact that a cluster has on its binary population. Here we summarize results of our search for X-ray-emitting binary stars in $\omega$ Cen using Chandra X-ray Observatory and Hubble Space Telescope (HST). The Chandra results have been reported by Haggard et al. [12] and the complete HST results will appear in Cool et al. [22]. 


\section{CHANDRA OBSERVATIONS AND RESULTS}

The Chandra observations were made using the Advanced CCD Imaging Spectrometer (ACIS), whose field of view (FOV) is $\sim 17^{\prime} \times 17^{\prime}$. For comparison, the half-mass radius of $\omega$ Cen is $4.2^{\prime}$ [23] and its core radius is $r_{c}=2.6^{\prime}$ [24]. With a total exposure of $\sim 70 \mathrm{ksec}$, we detected 180 sources to a limiting X-ray flux of $\sim 4.3 \times 10^{-16} \mathrm{erg} \mathrm{cm}^{-2} \mathrm{~s}^{-1}$. At the distance of $\omega$ Cen $(4.9 \mathrm{kpc})$, this corresponds to $L_{x} \sim 1.2 \times 10^{30} \mathrm{erg} \mathrm{sec}^{-1}$. Because $\omega$ Cen is very large on the sky, X-ray sources anywhere in the ACIS-I field can potentially be cluster members. However, given the large FOV and faint limiting flux of the observations, significant numbers of AGN will be present. After a statistical accounting of AGN as well as foreground stars, we estimated that $45-70$ of the Chandra sources are associated with the cluster. Based on nine optical IDs we projected that perhaps $20-35$ of the sources were cataclysmic variables (CVs), with most of the remainder being binaries containing coronally-active stars [see 12, for details].

Figure 1 shows an X-ray color-magnitude diagram for all the Chandra sources for which counts were recorded in three bands: "soft" $(0.5-1.5 \mathrm{keV})$, "medium" $(0.5-4.5 \mathrm{keV})$, and "hard" $(1.5-6.0 \mathrm{keV})$. Black symbols indicate the radial offset of each source from the cluster center; colored symbols mark X-ray sources for which optical identifications have been obtained. In Fig. 1 and the descriptions below, we exclude sources for which the identifications suggest they are not associated with the cluster (several AGN and a few foreground stars). We also exclude objects whose optical signatures are ambiguous. The complete set of optical IDs will be presented by Cool et al. [22].

\section{HST OBSERVATIONS AND ANALYSIS}

The HST data consist of 9 pointings with the ACS Wide Field Camera (WFC) covering $\sim 10^{\prime} \times 10^{\prime}$, approximately centered on the cluster; this field encompasses 109 of the Chandra sources. At each pointing we obtained four F625W $\left(\mathrm{R}_{625}\right)$, four $\mathrm{F} 435 \mathrm{~W}\left(\mathrm{~B}_{435}\right)$, and four $\mathrm{F} 658 \mathrm{~N}(\mathrm{H} \alpha)$ exposures. The broad-band exposures include one short exposure to measure the bright stars. To map the positions of Chandra sources onto the ACS/WFC images, we corrected the WFC images for distortion and then constructed mosaics in each filter. We used the star lists of Kaluzny et al. [25] and van Leeuwen et al. [26] to map R.A. and Dec. onto the mosaic images and then back onto the original "flt"-format images.

We did the photometric analysis using DAOPHOT/ALLSTAR [27] and ALLFRAME [28] on the "flt"-format images. We first extracted $\sim 20^{\prime \prime} \times 20^{\prime \prime}$ "patches" centered on each X-ray source from each of the ACS/WFC exposures available at the corresponding position. For most sources, this meant analyzing a total of 12 images. Because of the possibility that an interesting optical counterpart could be missed in a fully automated process, we carefully scrutinized the error circle region and iterated several times to insure that all objects identified within it were real and nothing was missed [details in 22]. Initially we adopted 1".0-radius error circles. Once several identifications had been made we performed a boresight correction which enabled us to reduce the radius to $0^{\prime \prime} 6$. Finally, we constructed $B_{435}-R_{625}$ vs. $R_{625}$ and $H \alpha-R_{625}$ vs. $R_{625}$ color-magnitude diagrams (CMDs) for each patch.

Once we had constructed CMDs for the patch around each X-ray source, we carried out a systematic evaluation of potentially interesting objects. All objects that did not lie on or very near the main sequence or giant branch in both the $B_{435}-R_{625}$ and $H \alpha-R_{625}$ CMDs were considered potentially interesting and evaluated for reliability of the photometry. We examined them in all the individual images to check the potential impact of near neighbors, cosmic rays, and diffraction spikes, and checked how cleanly DAOPHOT removed them from each of the images. We also took account of the consistency of multiple independent measurements in each filter. We then assigned a numerical index to represent the quality of the photometry $(0=$ best, $3=$ worst $)$; here we report only quality 0 and 1 candidates.

Figure 2 shows a combined $B_{435}-R_{625}$ vs. $R_{625}$ and $H \alpha-R_{625}$ vs. $R_{625}$ CMD for 35 of the optical IDs obtained using the HST data. Black dots mark the stars in the error circles of these 35 objects. To better delineate the turnoff and giant branch we also plot stars brighter than $R_{625}=19$ from the full $20^{\prime \prime} \times 20^{\prime \prime}$ patch associated with the qLMXB.

\section{OPTICAL COUNTERPARTS}

\section{Accreting Binary Stars: Cataclysmic Variables and a qLMXB}

Thirteen of the X-ray sources have an optical counterpart that is both blue and $\mathrm{H} \alpha$-bright (Fig. 2). This combination of signatures is strongly indicative of a compact accreting binary, with the $\mathrm{H} \alpha$ excess attributable to an emission line 


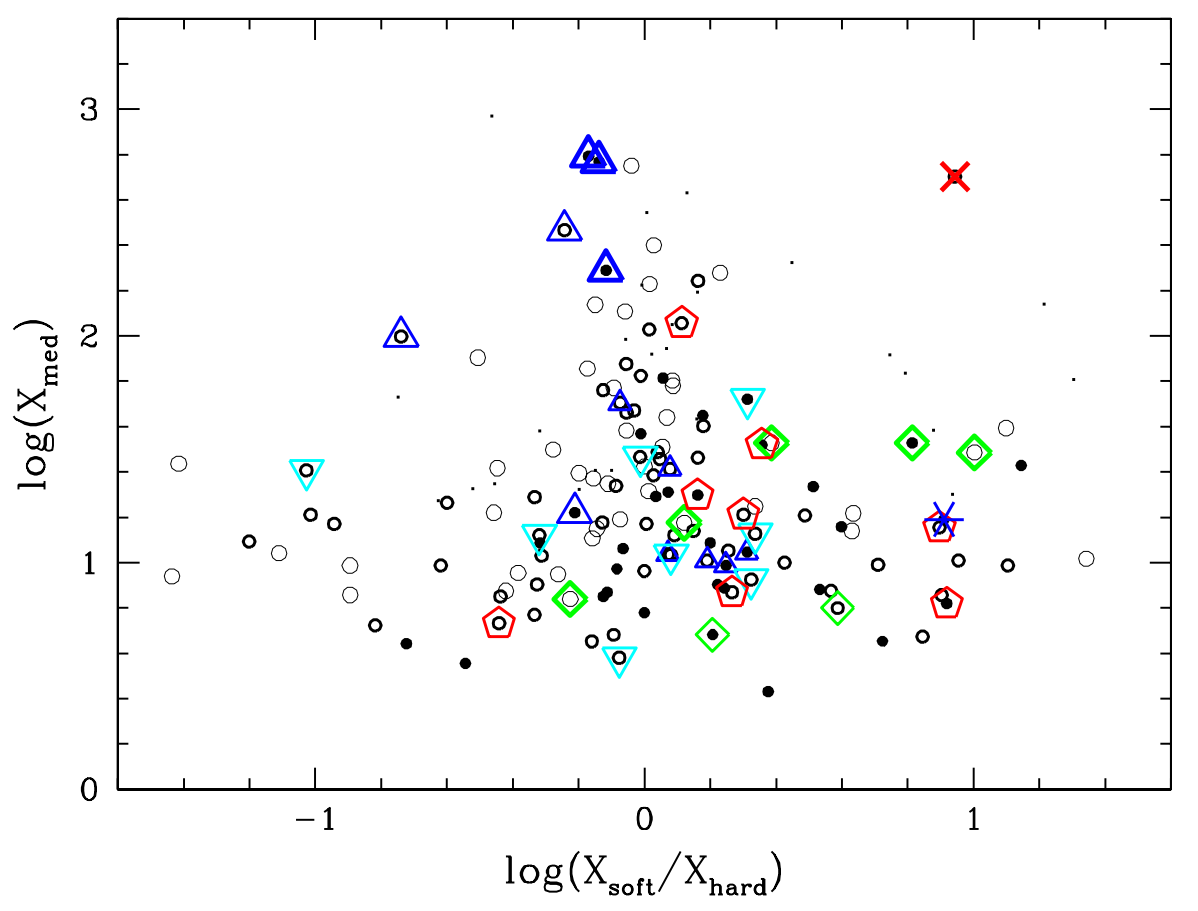

FIGURE 1. X-ray "color-magnitude" diagram for 164 Chandra sources in $\omega$ Cen with non-zero counts in both hard and soft bands. Round symbols indicate where in the cluster a source lies (large solid dots $=$ core; large open circles $=1-2 \mathrm{r}_{c}$; small open circles $=2-3 \mathrm{r}_{c}$; dots $=$ outside $3 \mathrm{r}_{c}$ ). Special symbols indicate optical IDs: a quiescent neutron star (red cross); previously known and newly identified CVs (large blue triangles); less certain new CVs (smaller blue triangles); very faint CV candidates with no H $\alpha$ detection (cyan inverted triangles); active binaries, e.g. BY Dra systems (green diamonds); possible sub-subgiants, also sometimes called red stragglers (red pentagons); and a candidate blue straggler (blue asterisk). Bold symbols indicate optical counterparts identified by Carson et al. [29] and Haggard et al. [12], including 5 ABs from the Kaluzny et al. [30] variable star catalog.

from an accretion disk and the blueness to the disk and/or the white dwarf. One of these is the object reported by Haggard et al. [31], which was first identified as a qNS on the basis of its X-ray spectrum [14]; it is marked with a red cross in Figures 1 and 2 . This is the only X-ray source in $\omega$ Cen with both the soft spectrum and the luminosity characteristic of a quiescent neutron star (see red cross in Fig. 1).

The remaining 12 optical counterparts that are both blue and $\mathrm{H} \alpha$-bright have X-ray colors and luminosities typical of cataclysmic variables (CVs; see blue triangles). Three of these (bold blue triangles) were known from previous studies $[29,31]$; nine are new. The best of the new candidates (large blue triangles) are all easily confirmed visually as being blue and all individual $H \alpha-R_{625}$ measurements are consistent in showing that they are $\mathrm{H} \alpha$-bright. The remaining six $\mathrm{CV}$ candidates (small blue triangles) are visually confirmed as either blue or $\mathrm{H} \alpha$-bright but have somewhat lower confidence associated with either their $\mathrm{H} \alpha$ excess or blueness. Nevertheless, we think it probable that most, if not all, of these stars are the optical counterparts of the X-ray sources and are CVs.

In addition to these $12 \mathrm{CV}$ candidates, we find very faint blue stars in the error circles of nine of the Chandra sources (see inverted cyan triangles). These nine stars are exceedingly faint $\left(R_{625}=24.5-26.3\right)$. Most are seen in $B_{435}$ only because they are so blue; main-sequence stars of comparable $R_{625}$ magnitude are below the detection limit. All but one were confirmed visually as being blue by blinking $R_{625}$ vs. $B_{435}$ images. However, as none of these stars are detected in $\mathrm{H} \alpha$, they do not appear in the $H \alpha-R_{625}$ diagram (see Fig. 2). Given that these stars lie in the region of the CMDs generally occupied by white dwarfs (WDs), we have considered the possibility that they could be WDs that have landed by chance in the Chandra error circles and are unrelated to the X-ray sources. Our statistical analysis [22] suggests that perhaps one could be such a chance coincidence, but that it is unlikely that many more could be explained in this way. We therefore suggest that these objects are also CVs. It is important to note that the lack of $\mathrm{H} \alpha$ detections does not necessarily imply that they are not $\mathrm{H} \alpha$-bright; they may simply be too faint to be detected, even in the presence of an $\mathrm{H} \alpha$ emission line. 


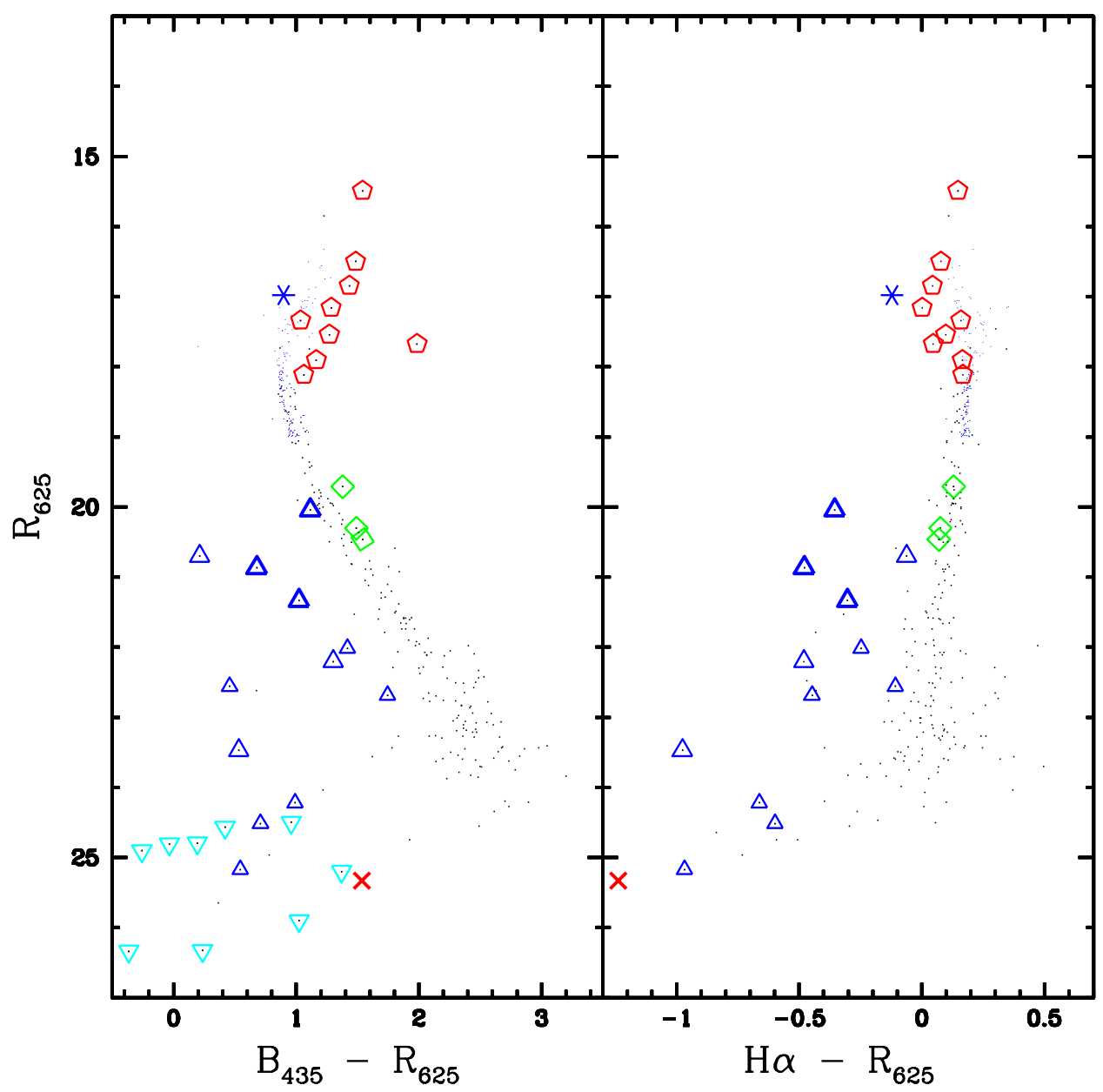

FIGURE 2. Color-magnitude diagram in $\mathrm{B}_{435}-\mathrm{R}_{625}$ vs. $\mathrm{R}_{625}$ (left panel) and $\mathrm{H} \alpha-\mathrm{R}_{625}$ vs. $\mathrm{R}_{625}$ (right panel) showing all stars that appear in the error circles of $35 \mathrm{X}$-ray sources for which promising optical identifications have been made. Black dots represent all the stars in the error circles. To better delineate the turnoff region and red giant branch, all stars with $\mathrm{R}_{625}<19$ in the full $20^{\prime \prime} \times$ $20^{\prime \prime}$ patch around the qLMXB are shown as blue dots. Suggested optical counterparts of X-ray sources are marked with symbols as in Fig. 1. Note that the 5 ABs obtained by matching X-ray sources to the Kaluzny et al. [30] variable star catalog are not shown as they either lie outside the HST FOV or were not distinctive in the HST data.

\section{Binaries Containing Stars with Active Coronae}

X-ray imaging with Chandra of nearby GCs is also sensitive enough to pick up binaries containing coronally active stars. Falling broadly into this category are the five optical identifications by Haggard et al. [12] based on comparison of the variable star catalog of Kaluzny et al. [30] with the Chandra source list. These five sources, which include two eclipsing Algols and a long-term variable, are shown as bold green diamonds in Figure 1. They do not appear in Figure 2, as four are outside the HST FOV, and the fifth was not picked up as being distinctive in the HST CMDs.

Narrow-band imaging with $\mathrm{H} \alpha$ also enables us to search for the elevated levels of coronal activity associated with certain types of binary stars (e.g., BY Dra and RS CVn). In the field, this activity is typically the result of fast spin rates induced by tidal synchronization [see 32, and references therein]. However, H $\alpha$ equivalent widths for such stars are much lower than for CVs, $\sim 1-3$ Angstroms [33]. Given the $\sim 80$ Angstrom width of the ACS/WFC H $\alpha$ filter, such stars will appear only very slightly $\mathrm{H} \alpha$-bright (at best) in the present study. To limit the number of false positives in our search, we required that a star show both an $\mathrm{H} \alpha$ signature and also be above the main sequence in the individual $B_{435}-R_{625} \mathrm{CMD}$ associated with its patch. The latter requirement excludes binaries whose mass ratio is much less than unity. The three candidate BY Dra stars we found in this way are shown in Fig. 2 (green diamonds). In Fig. 1 it can be seen that, on average, these are relatively faint and moderately soft X-ray sources. 


\section{A Blue Straggler or Turnoff Binary?}

One of the candidates (blue asterisk in Fig. 2) appears above the turnoff, to the blue side of the subgiant branch, a location which is suggestive of a blue straggler. The star has a 0.25 -magnitude $\mathrm{H} \alpha$ excess, which strongly suggests that it is the X-ray source. However, the star also lies $\lesssim 0.75$ magnitudes above the turnoff and thus may instead be a detached binary containing two turnoff stars. We suggest that this star probably falls in the broad category of active stars, in the sense that its X-ray emission is most likely to be associated with an active corona. However, we give it its own symbol in the CMDs to distinguish it as a special case.

\section{Sub-subgiants or Anomalous RGB Stars?}

Nine of the candidates we have identified appear to the red side of the main-sequence turnoff and subgiant and giant branches (Fig. 2; red pentagons). Several show signs of $\mathrm{H} \alpha$ in emission, which is strongly associated with enhanced $\mathrm{X}$-ray emission and supports an association between these stars and the X-ray sources. Given their location in the $\mathrm{CMD}$, we tentatively identify these stars as sub-subgiants [SSG; 34], also sometimes called red stragglers. However, a close inspection of the CMD shows that 7 of the stars lie along the metal-rich "anomalous" red giant branch [35, 19]. Thus it is possible that these stars are instead a subset of the anomalous RGB stars which for some reason are unusually $\mathrm{X}$-ray bright.

\section{DISCUSSION}

Using a combination of Chandra and HST imaging in blue, red and $\mathrm{H} \alpha$ filters, we have identified a total of $40 \mathrm{X}$-rayemitting binary stars in $\omega$ Cen. Five were found as X-ray counterparts of variable stars reported by Kaluzny et al. [30] and four had been found in earlier Chandra and/or HST studies. The remaining 31 are newly reported here.

Accreting binary stars make up just over half of the identifications: one qLMXB and 21 candidate CVs. The remaining identifications are evenly split into two broad classes: 9 active binaries and 9 objects that are possible sub-subgiants. The active binaries include an assortment of different types of systems, all of whose X-ray emission is likely due to active coronae. These include two eclipsing Algol systems, three possible BY Dra stars, and a blue straggler.

Of particular interest among the CV candidates are the nine very faint blue stars shown as inverted triangles in Figures 1 and 2. Given the distance modulus to $\omega$ Cen, their absolute magnitudes are in the range $M_{625}=10.9-12.7$. This is comparable to the absolute magnitudes of the short-period CVs recently uncovered in the Sloan Digital Sky Survey [SDSS; 36]. Thus the systems in $\omega$ Cen could be short-period systems with very low-mass secondaries, as is expected for very old CVs. Their positions in the X-ray CMD (see Fig. 1) generally support the CV interpretation. Alternatively, some of these objects could be helium white dwarfs with MSP companions; a few such systems are known in globular clusters [e.g. 37]. Deeper $\mathrm{H} \alpha$ imaging and/or multiwavelength broad-band imaging is needed to distinguish between these possibilities.

Given that the faintest CVs detected in this study are at the detection limit in the both the X-ray and optical images, it is likely that more CVs remain to be discovered in $\omega$ Cen. How many more depends on the relative numbers of faint vs. bright $\mathrm{CVs}$ - a ratio that depends both on the evolution of CVs and their formation history in the cluster. The present study shows that even very faint CVs can be found in the crowded environs of $\omega$ Cen; deeper observations should allow a more complete census to be made.

The present census of active binaries in $\omega$ Cen is undoubtedly very incomplete. At X-ray wavelengths we are likely seeing just the tip of the iceberg. In the optical, we are hampered by the weakness of the $\mathrm{H} \alpha$ emission lines. Observations with a narrower $\mathrm{H} \alpha$ filter can help, as demonstrated by the large number of BY Dra stars identified by Taylor et al. [38] in NGC 6397 using a 20 Angstrom-wide filter.

Perhaps the most intriguing set of X-ray-emitting stars identified in this study are the nine that lie redward of the turnoff and giant branch. While their close proximity to the evolutionary sequences in $\omega$ Cen suggests that they are associated with the cluster, proper motions are needed to be sure. If spectroscopic observations reveal that some or all are members of the anomalous RGB, then it will be important to understand why this subpopulation in $\omega$ Cen is prone to producing X-ray-bright stars. If instead these stars turn out to be bonafide SSGs, then $\omega$ Cen should provide a valuable testing ground for studying this as-yet poorly understood class of X-ray-emitting binary systems. 


\section{REFERENCES}

1. J. M. Fregeau, and F. A. Rasio, ApJ 658, 1047-1061 (2007), arXiv:astro-ph/ 0608261.

2. N. Ivanova, C. O. Heinke, F. A. Rasio, R. E. Taam, K. Belczynski, and J. Fregeau, MNRAS 372, 1043-1059 (2006), arXiv:astro-ph/0604085.

3. J. M. Fregeau, M. A. Gürkan, K. J. Joshi, and F. A. Rasio, ApJ 593, 772-787 (2003), arXiv : astro-ph/0301521.

4. D. Pooley, and P. Hut, ApJL 646, L143-L146 (2006), arXiv: astro-ph/ 0605048.

5. J. M. Fregeau, ApJL 673, L25-L28 (2008), arXiv:astro-ph/ 0710.4556.

6. E. F. Brown, L. Bildsten, and R. E. Rutledge, ApJL 504, L95 (1998), arXiv: astro-ph/ 9807179.

7. C. O. Heinke, J. E. Grindlay, D. A. Lloyd, and P. D. Edmonds, ApJ 588, 452-463 (2003), arXiv : a stro-ph/0301235.

8. C. O. Heinke, J. E. Grindlay, P. M. Lugger, H. N. Cohn, P. D. Edmonds, D. A. Lloyd, and A. M. Cool, ApJ 598, 501-515 (2003), arXiv:astro-ph/0305445.

9. D. Pooley, W. H. G. Lewin, F. Verbunt, L. Homer, B. Margon, B. M. Gaensler, V. M. Kaspi, J. M. Miller, D. W. Fox, and M. van der Klis, ApJ 573, 184-190 (2002), arXiv: astro-ph/0111212.

10. C. O. Heinke, J. E. Grindlay, P. D. Edmonds, H. N. Cohn, P. M. Lugger, F. Camilo, S. Bogdanov, and P. C. Freire, ApJ 625, 796-824 (2005), arXiv:astro-ph/0503132.

11. P. M. Lugger, H. N. Cohn, C. O. Heinke, J. E. Grindlay, and P. D. Edmonds, ApJ 657, 286-301 (2007), arXiv: astro-ph/0606382.

12. D. Haggard, A. M. Cool, and M. B. Davies, ApJ 697, 224-236 (2009), arXiv: astro-ph/ 0902.2397.

13. D. Pooley, W. H. G. Lewin, L. Homer, F. Verbunt, S. F. Anderson, B. M. Gaensler, B. Margon, J. M. Miller, D. W. Fox, V. M. Kaspi, and M. van der Klis, ApJ 569, 405-417 (2002), arXiv: astro-ph/ 0110192.

14. R. E. Rutledge, L. Bildsten, E. F. Brown, G. G. Pavlov, and V. E. Zavlin, ApJ 578, $405-412$ (2002), arXiv: astro-ph/0105405.

15. G. Meylan, "The Globular Cluster $\omega$ Centauri: A General Overview," in Omega Centauri, A Unique Window into Astrophysics, edited by F. van Leeuwen, J. D. Hughes, \& G. Piotto, 2002, vol. 265 of Astronomical Society of the Pacific Conference Series, pp. 3.

16. L. R. Bedin, G. Piotto, J. Anderson, S. Cassisi, I. R. King, Y. Momany, and G. Carraro, ApJL 605, L125-L128 (2004), arXiv:astro-ph/0403112.

17. R. Gratton, C. Sneden, and E. Carretta, ARA\&A 42, 385-440 (2004).

18. G. Piotto, S. Villanova, L. R. Bedin, R. Gratton, S. Cassisi, Y. Momany, A. Recio-Blanco, S. Lucatello, J. Anderson, I. R. King, A. Pietrinferni, and G. Carraro, ApJ 621, 777-784 (2005), arXiv: astro-ph/ 0412016.

19. S. Villanova, G. Piotto, I. R. King, J. Anderson, L. R. Bedin, R. G. Gratton, S. Cassisi, Y. Momany, A. Bellini, A. M. Cool, A. Recio-Blanco, and A. Renzini, ApJ 663, 296-314 (2007), arXiv: astro-ph/0703208.

20. E. Noyola, K. Gebhardt, and M. Bergmann, ApJ 676, 1008-1015 (2008), arXiv: astro-ph/0801.2782.

21. J. Anderson, and R. P. van der Marel, ApJ 710, 1032-1062 (2010), arXiv : astro-ph/0905.0627.

22. A. M. Cool, D. Haggard, T. Arias, M. Brochmann, J. Dorfman, M. V. White, and J. Anderson, in preparation (2010).

23. W. E. Harris, VizieR Online Data Catalog 7195, 0 (1996).

24. S. C. Trager, I. R. King, and S. Djorgovski, AJ 109, 218-241 (1995).

25. J. Kaluzny, M. Kubiak, M. Szymanski, A. Udalski, W. Krzeminski, and M. Mateo, A\&AS 120, 139-152 (1996), arXiv:astro-ph/9601053.

26. F. van Leeuwen, R. S. Le Poole, R. A. Reijns, K. C. Freeman, and P. T. de Zeeuw, A\&A 360, 472-498 (2000).

27. P. B. Stetson, PASP 99, 191-222 (1987).

28. P. B. Stetson, PASP 106, 250-280 (1994).

29. J. E. Carson, A. M. Cool, and J. E. Grindlay, ApJ 532, 461-466 (2000).

30. J. Kaluzny, A. Olech, I. B. Thompson, W. Pych, W. Krzemiński, and A. Schwarzenberg-Czerny, A\&A 424, 1101-1110 (2004), arXiv:astro-ph/0406456.

31. D. Haggard, A. M. Cool, J. Anderson, P. D. Edmonds, P. J. Callanan, C. O. Heinke, J. E. Grindlay, and C. D. Bailyn, ApJ 613, 512-516 (2004), arXiv: astro-ph/0312657.

32. V. V. Makarov, and P. P. Eggleton, ApJ 703, 1760-1765 (2009).

33. A. Young, F. Ajir, and G. Thurman, PASP 101, 1017-1031 (1989).

34. R. D. Mathieu, M. van den Berg, G. Torres, D. Latham, F. Verbunt, and K. Stassun, AJ 125, $246-259$ (2003), arXiv:astro-ph/0209568.

35. E. Pancino, F. R. Ferraro, M. Bellazzini, G. Piotto, and M. Zoccali, ApJL 534, L83-L87 (2000), arXiv: astro-ph/ 0003222 .

36. B. T. Gänsicke, M. Dillon, J. Southworth, J. R. Thorstensen, P. Rodríguez-Gil, A. Aungwerojwit, T. R. Marsh, P. Szkody, S. C. C. Barros, J. Casares, D. de Martino, P. J. Groot, P. Hakala, U. Kolb, S. P. Littlefair, I. G. Martínez-Pais, G. Nelemans, and M. R. Schreiber, MNRAS 397, 2170-2188 (2009), arXiv: astro-ph/ 0905 . 3476.

37. P. D. Edmonds, R. L. Gilliland, C. O. Heinke, J. E. Grindlay, and F. Camilo, ApJL 557, L57-L60 (2001), arXiv:astro-ph/0107096.

38. J. M. Taylor, J. E. Grindlay, P. D. Edmonds, and A. M. Cool, ApJL 553, L169-L172 (2001). 